\title{
A Phased Array Acoustic Wind Profiler for Remote Atmospheric Wind Measurement
}

\author{
Madhu Bahl, Beena Gupta, Thomas John, Dhan Singh, Omkar Sharma, S. C. Garg and Ravi M. Khanna
}

National Physical Laboratory, Dr. K. S. Krishanan Road, New Delhi - 110 012, India

\begin{abstract}
A phased array acoustic atmospheric wind profiler has been developed. The system has the capability to remotely measure atmospheric horizontal and vertical winds from $70 \mathrm{~m}$ to $770 \mathrm{~m}$ within the earth's atmospheric boundary layer. This system is being operated at the National Physical Laboratory (NPL), which is located in the middle of a highly noisy environment of the city of Delhi. Moreover, due to the rich green surroundings, there are plenty of birds whose calls make measurements at acoustic frequencies very difficult. In spite of the above two limitations, the system has been successfully used to make wind measurements in the height range of $70 \mathrm{~m}$ to $455 \mathrm{~m}$.
\end{abstract}

\section{Keywords:}

Acoustic, Antenna, Atmosphere, Noise, Profiler, Wind.

\section{INTRODUCTION}

Wind measurement in the earth's lower atmosphere has powerful emerging applications. Since long, wind measurement has almost been an exclusive domain of the meteorologist, who needed wind information to generate weather forecasts. This information has since long been obtained using in situ sensors mounted either on fixed platforms such as meteorological towers or mobile platforms such as tethered balloons. Since the advent of radars, about 6-7 decades ago, wind information has also been obtained using freely drifting balloons that are tracked by them. These methods, although good in terms of accuracy, do not offer temporal and range continuity of measurement, as well as, the range resolutions that the technological applications of the time demand [1]. Recent developments in remote sensing technology, using radio, light and sound waves, have overcome many of the limitations of the conventional techniques of wind measurement. Among the remote sensing techniques, when it comes to obtaining wind patterns in the earth's lower atmospheric layer, there is no better option than the acoustic wind profiler. Such a system using a single compact phased array antenna has been developed and used to make atmospheric wind measurements.

\section{PRINCIPLE OF WIND MEASUREMENT USING AN ACOUSTIC WIND PROFILER}

The interface between the earth and its atmosphere, which is generally called the earth's boundary layer, is rich in turbulent inhomogeneities of wind and temperature or turbulent eddies, as these are generally called. These turbulent eddies are generated by thermal and mechanical processes caused by differential heating of the earth's surface as well as due to obstacles over the earth. These eddy or parcels of air that are of varying sizes ride on winds and drift along with their movement. Measurement of movement of these eddies has, here, been used as a means for indirect measurement of the movement of the winds.

When a pulse of sound is radiated into the atmosphere, it interacts with these eddies and the energy in the sound pulse is scattered in all directions. The Doppler shift produced in the backscattered signals due to the movement of these eddies is measured and computations are made to derive radial winds that are then used to give horizontal and vertical winds.

The backscattered electrical power $\mathrm{P}_{\mathrm{r}^{\prime}}$ at the input of the receiver, obtained from any scattering volume located at range $\mathrm{R}$ from the antenna that has an effective aperture $\mathrm{A}_{\mathrm{e}}$, has been given by Little [2] as

$\mathrm{P}_{\mathrm{r}}=\mathrm{P}_{\mathrm{t} .} \sigma c \tau \mathrm{A}_{\mathrm{e}} / 2 \mathrm{~L} \mathrm{R}^{2}$

In equation (1), $\mathrm{P}_{\mathrm{t}}$ is the transmitted sound power of the pulsed sinusoidal wave of pulse width $\tau$ in seconds, traveling with a velocity $\mathrm{c}$. $\mathrm{L}$ in the above equation is a dimensionless loss factor that includes both the to and fro attenuation of the sound wave from antenna to scattering volume, as well as, the acoustic to electrical conversion inefficiency and $\sigma$ is the backscattering cross section per unit volume per unit solid angle of the scattering volume.

The scattering of sound waves by both temperature and velocity fluctuations has been treated by Monin [3], with experimental checks to the theory of sound 
scattering provided by Kallistratova [4,5]. In the backscatter mode of operation of the system, i.e. the scheme that we have adopted, where the antenna that transmits the sound pulse acts in reverse to receive signals during the receive cycle of system operation, it can be seen that the contribution to the scattered signals is only due to temperature turbulence, and is given by the scattered power $\sigma$ per unit volume, per unit incident flux, per unit solid angle as

$\sigma=0.0072\left(\mathrm{C}_{\mathrm{T}}^{2} / \mathrm{T}^{2}\right)(1 / \lambda)^{1 / 3}$

In equation (2), $\mathrm{C}_{\mathrm{T}}{ }^{2}$ is the structure parameter of the temperature turbulence, $\mathrm{T}$ is the mean temperature of the scattering volume and $\lambda$ is the wave length of the transmitted signals.

The sound waves illuminating the scatterers in the spatial scattering volume generate a Doppler spectrum due to the motion of the distributed targets, which may be expressed as Doppler shifts $\Delta \mathrm{f}_{\mathrm{i}}, \mathrm{i}=1$ to $n$, where $\Delta \mathrm{f}_{\mathrm{i}}$ is the Doppler shift of any $\mathrm{i}^{\text {th }}$ individual scatterer in the volume and $n$ is the total number of scatterers. Each of the Doppler spectral shift $\Delta \mathrm{f}_{\mathrm{i}}$ corresponds to velocity $\mathrm{v}_{\mathrm{i}}$ of the scatterer in the scattering volume according to the Doppler equation, $v_{i}=-c / 2\left(\Delta f_{i} / f\right)$, where $f$ is the transmitted frequency. The above equation assumes that the motion of the scatterers away from the antenna is positive. Then, the resulting wind velocity, at any height range, is a volume-averaged estimate or vector sum of the velocities of all the scatterers within the resolution volume and is thus derived from the received spectrum.

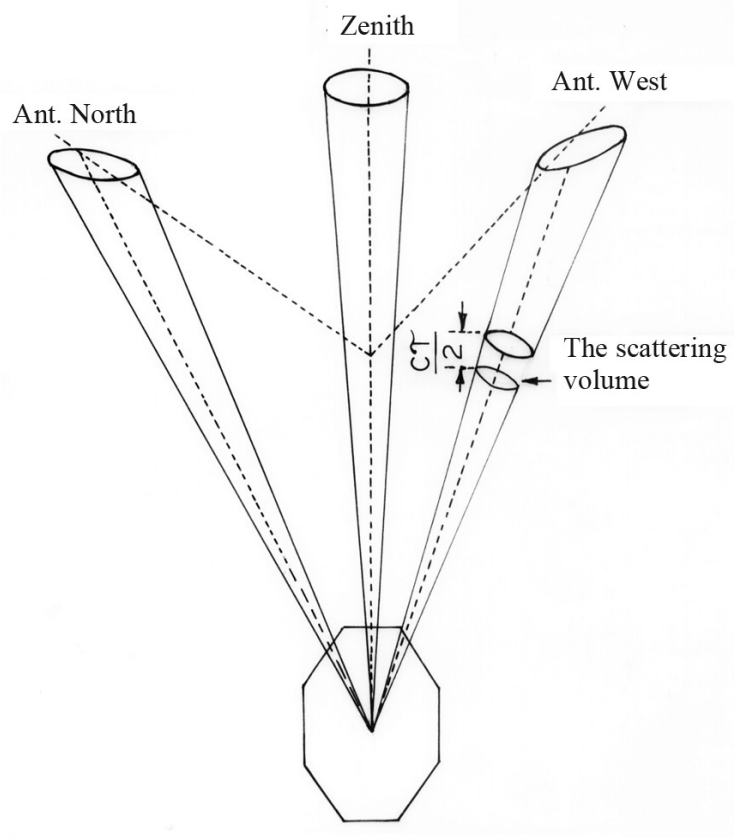

Acoustic phased array antenna

Figure 1: Acoustic phased array antenna.

\section{METHODOLOGY AND SYSTEM}

The acoustic wind profiler has been developed around an indigenously designed single flat, compact piezoelectric elements phased array antenna. The antenna generates a powerful acoustic beam that has the capability to be electronically steered in three atmospheric directions as shown in Figure 1, for threedimensional measurements of atmospheric winds. The antenna beam sequentially points toward zenith and two near orthogonal orientations that are off zenith by $19^{\circ}$, in both the transmit, as well as, the receive modes of system operation. A block schematic of the acoustic wind profiler is given in Figure 2. For the purpose of descriptive clarity, the system may be divided into the following six subsystems:

(a) Phased array antenna

(b) Switching matrix

(c) Transmitter

(d) Receiver

(e) Data acquisition and processing

(f) Timing and control

A brief description of each of the subsystem follows.

\section{(a) Phased Array Antenna}

The heart of the wind profiler is a high-efficiency phased array acoustic antenna designed using 104 numbers of KSN1025A piezoelectric transducers (Motorola Inc., Schaumvburg, Illinois, USA). The adjoining elements of the antenna are electrically combined to convert it into a 52-coupled element antenna for the sake of simplicity, as well as, element dimensional requirement for inter-row or inter-column spacing required for the steering of the acoustic beam, as these 52 elements are then configured as 11 rows or 11 columns of the antenna. All the rows or columns do not have the same number of elements. The number of elements in each row or column is tapered

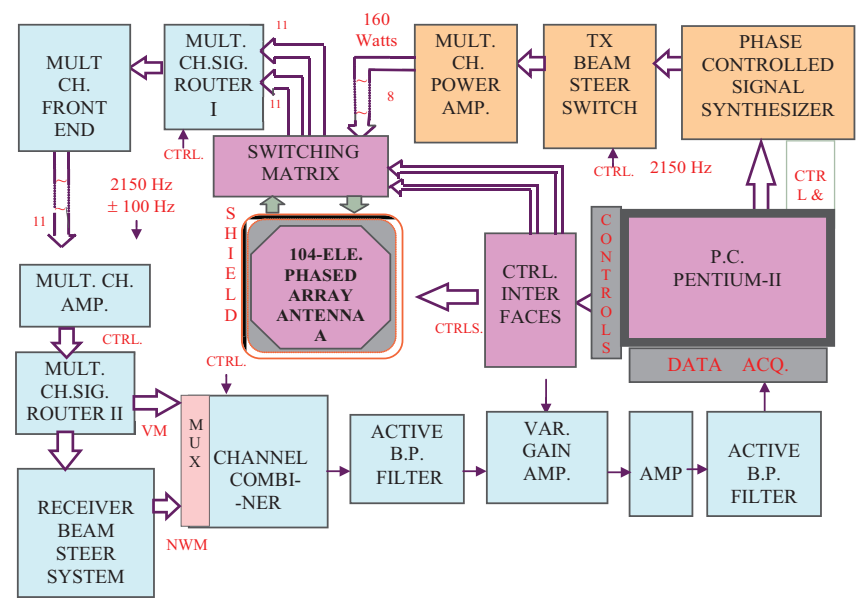

Figure 2: A block schematic of the phased array acoustic wind profiler. 
from the center to the ends to improve the directional characteristics of the acoustic beam.

The antenna, after fabrication, was subjected to rigorous analysis in respect of its transmit and receive efficiencies, as well as, directional characteristics, in the NPL Acoustic Anechoic Chamber. The transmit efficiency of the antenna at the system operating frequency of $2150 \mathrm{~Hz}$ is $14.5 \mathrm{~Pa}$ of acoustic pressure (obtained at $1 \mathrm{~m}$ distance from the antenna acoustic center along the antenna acoustic axis) per volt of rms signal applied to it, whereas its receive efficiency at the same frequency is $0.7 \mathrm{VPa}^{-1}$ of acoustic pressure applied at its aperture. It is observed that the conversion gain factor (product of the above two efficiencies) of around 10 obtained in this antenna is greater than around more than two orders of magnitude compared with the paraboloidal dish antenna (used in older systems) of comparable dimensions [6].

The antenna, during operation, is enclosed in an acoustic shield lined with a $50 \mathrm{~mm}$ thick medium-density foam to suppress its side lobes, as well as, to reduce the surrounding acoustic noise from entering into it, which otherwise reduces the signal to noise ratio of the received signal. Figure 3 depicts a view of the antenna placed inside the acoustic shield. The design and evaluation of the array antenna in respect of its transmit and receive efficiencies, as well as, its beam patterns are dealt with elsewhere [7].

\section{(b) Switching Matrix}

In the transmit mode of system operation, signals from the transmitter to the antenna, as well as, from the antenna to the receiver, in the receive mode of its

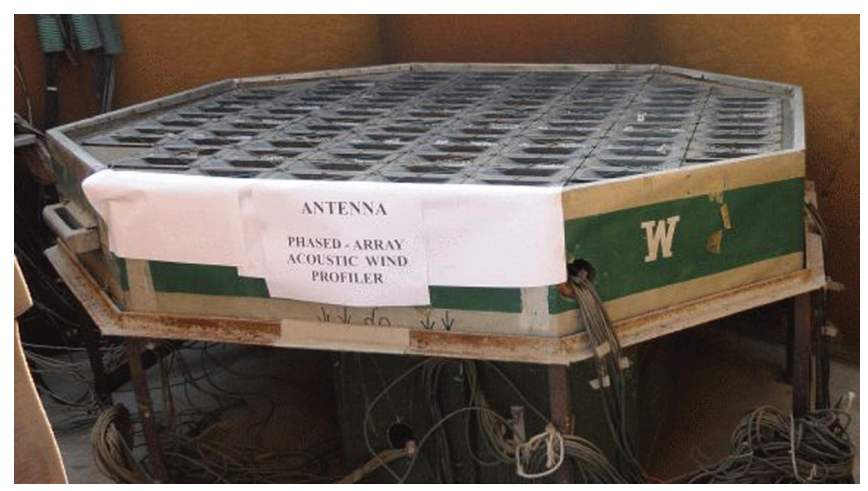

Figure 3: A view of the phased array acoustic antenna inside the acoustic shield, at the site of the experiment. operation, are routed via a complex Transmit/ReceiveRow/Column (TR-RC) switching matrix. This switching matrix is integrated at the back of the phased array antenna. This subsystems is designed using 104 numbers of GA6A electro- mechanical relays (Omron, Minato-ku, Tokyo, 108-0075 JAPAN). These relays configure the elements of the array into different groups for the transmitter to pump electrical power into the antenna. These relays of the switching matrix also configure the coupled elements of the antenna into different rows and columns for reception, respectively, from the antennanorth or antenna-west steered atmospheric directions. The switching functions of the matrix are obtained on application of different control signals generated at appropriate times by the control software of the system. The antenna-north and antenna-west are the conventions adopted to designate the steering directions of the antenna beam that are then aligned with geographical directions.

\section{(c) Transmitter}

The transmitter comprises of three subsystems: (1) a phase-controlled signal synthesizer, (2) a transmit beam steering switch and (3) a bank of power amplifiers. The signal synthesizer generates four signals, all at a frequency of $2150 \mathrm{~Hz}$, which are either in phase or at a phase spacing $\Delta \phi$ of $\pi / 2$, i.e. at phases of $-\pi / 2,0, \pi / 2$ and $\pi$. These signals are then input to the transmit beam steering switch that outputs eight channels of signals that are either in phase or at two different sets of phase relationships.

These eight channel signals are sufficient to give a phase taper of $\pi / 2$ to 11 rows or 11 columns of the array antenna. The eight channel signals are power-amplified in the power amplifier bank and fed to eight antenna groups of elements with phases as given in Table 1. Now, when the eight antenna groups are excited with in-phase signals, the antenna radiates an acoustic beam in the vertical direction. When the antenna groups are fed with a set of phase-related signals as given in Table 1 , as north steering mode, the rows of the antenna are excited with signals at a phase spacing of $\pi / 2$, and the acoustic beam of the antenna gets steered by $19^{\circ}$ from vertical toward the antenna-north. On the other hand, when the antenna groups are fed with a second set of phase-related signals as given in Table 1, as west steering mode, the columns of the antenna are excited

Table 1: Antenna group's phase relationship for the three steering modes

\begin{tabular}{lcccccccc}
\hline $\begin{array}{l}\text { Phases for: } \\
\text { Steering mode: }\end{array}$ & Group A Deg & Group A Deg & Group A Deg & Group A Deg & Group A Deg & Group A Deg & Group A Deg & Group A Deg \\
\hline Vertical & 0 & 0 & 0 & 0 & 0 & 0 & 0 & 0 \\
North & 0 & 90 & 0 & -90 & 90 & 180 & -90 & 180 \\
West & 90 & 0 & -90 & 0 & 180 & 90 & 180 & -90 \\
\hline
\end{tabular}


with signals at a phase spacing of $\pi / 2$ and the acoustic beam of the antenna gets steered by $19^{\circ}$ from vertical toward antenna-west. These functions of the antenna are facilitated by the TR-RC matrix located at its back. If $\mathrm{d}$ is the periodic spacing between the adjacent rows or columns and $\lambda$ is the wavelength of the transmitted sound signals, then the steering angle $\theta$ is given by equation (3) as,

$\theta=\sin ^{-1}[(\lambda / d)(\Delta \phi / 2 \pi)]$

\section{(d) Receiver}

The acoustic signals that are radiated into the atmosphere interact with atmospheric turbulent inhomogeneities during their outward propagation and get scattered. Signals scattered back towards the acoustic antenna are picked up by this sensitive antenna and are converted to the respective 11 channels of electrical signals by the different rows or columns of the antenna. These back-scattered signals that are received from different height ranges are quite weak and, moreover, deeply embedded in noise. These are then input into a bank of low-noise, high-gain pre-amplifiers designed using INA 101 instrumentation amplifier (Texas Instruments Inc, Dallas, Texas 75243, USA) that have an input noise voltage of $13 \mathrm{nV} / \sqrt{ } \mathrm{Hz}$, with each of the amplifiers giving a gain of $78 \mathrm{~dB}$. However, signals to the pre-amplifier bank are routed via the multichannel signal router-I. This signal router selects signals either from the rows or from the columns, respectively, for the signal reception from the north-radial or west-radial directions. Low-noise, high-gain inputs limit the contribution due to electrical noise to negligible levels compared with the contribution due to the environmental acoustic noise, which also gets converted to electrical noise by the acoustic antenna and, which, in fact, becomes the limiting factor for the accuracy, as well as, the height of wind measurement as it affects the signal to noise ratio.

The above 11 channel signals are then input into a second bank of amplifiers designed using OP027 operational amplifiers (Analog Devices Inc., Norwood, MA,USA) that provide a gain of $26 \mathrm{~dB}$. The output of this bank is fed to the multi-channel signal router-II. This signal router either inputs these signals directly into the channels combiner for vertical wind measurement or via the receiver beam steering system for west-radial or northradial modes of wind measurement. As against the phase delay beam steering technique used in the transmitter, the receiver beam steering system uses the time delay beam steering technique as the latter is independent of frequency and, therefore, the steering angle remains unaffected by Doppler shift in the received signals.

The receiver beam steering system steers the antenna beam in the direction in which the sound pulse had been transmitted, i.e. turned $19^{\circ}$ from vertical toward antenna-north and antenna-west, respectively. This is done by generating incremental time delays $\Delta t$ between adjacent rows or columns of the array antenna. Then the receive beam steering angle $\theta$ of the antenna is given by equation (4) as,

$\theta=\sin ^{-1}[(\mathrm{c} / \mathrm{d}) \Delta \mathrm{t}]$

The periodic time delay in our case is around $116 \mu \mathrm{s}$. The suitably delayed 11 channel signals are then combined in the channel combiner.

The combined signals, either direct or steered modes, are input into an eighth-order Chebychev active band pass filter with a bandwidth of $\pm 75 \mathrm{~Hz}$, designed using UAF42 active filter ICs (Texas Instruments Inc, Dallas, Texas 75243, USA). This band pass filter rejects all noise that lies outside the band of the filter and the filtered signals are then passed through a range compensation amplifier that provides a variable gain of $20 \mathrm{~dB}$ for the different range gates to compensate for the attenuation of sound signals due to range. This is done using an analog multiplier that multiplies the received signals with stepped DC signals generated by the PC which correspond to each range gate. The variable gain is then scaled up by $6 \mathrm{~dB}$ by a fixed gain amplifier to bring the signals to levels acceptable for the data acquisition system. However, before the signals are input to the data acquisition unit in the PC, these are passed through a second stage of eighth order Chebychev filter to remove any noise generated in the multiplier stage.

\section{(e) Data Acquisition and Processing}

Signals, conditioned as above, are then sampled and digitized with 12-bit resolution by a data acquisition system and the time series of the digitized signals are generated, corresponding to each range gate. Initial blanking of the received signals, carried out to avoid measurement during the prolonged antenna vibrations due to transmission of high-transmit pulse, as well as, nearby building echoes, limits the lower range of wind measurement to $70 \mathrm{~m}$. Frequency domain spectra are generated for signal-plus-noise, as well as, for pure noise. This is done by receiving and processing the signals, with the transmitter radiating the pulse, as well as, receiving and processing the signals with the transmitter not radiating the pulse. The noise spectrum of the latter case is then subtracted from the signal plus-noise spectrum of the former to obtain the noise-cleaned signal spectrum.

We fixed the velocity spectral resolution as $0.5 \mathrm{~m} \mathrm{~s}^{-1}$. This corresponds to a frequency resolution (using the Doppler equation) of around $6.25 \mathrm{~Hz}$. We transmitted a 
pulse of $200 \mathrm{~ms}$. Therefore, the received signal is gated for $200 \mathrm{~ms}$. This period is divided between sampling and processing, assigning $160 \mathrm{~ms}$ for sampling and 40 ms for computation of the Fast Fourier Transform (FFT) spectrum. We need to sample at more than twice the signal frequency to avoid aliasing. Therefore, we sample at $6.4 \mathrm{kHz}$, which gives 1024 points in $160 \mathrm{~ms}$. However, we select only $n=24$ points around our center frequency of $2150 \mathrm{~Hz}$ to calculate the mean Doppler shift as given in the following equation. From the noise-corrected spectrum, corresponding to each range gate, the signal power $P$ is computed using equation (5) as,

$P=\sum_{i=1}^{n} P_{i}$

Where $\mathrm{P}_{\mathrm{i}}$ is the power of an individual spectral point and $n$ corresponds to the number of spectral points or width of the spectrum. Then, the mean Doppler shift $\Delta \mathrm{f}$ is given by equation (6) as,

$\Delta f=1 / P \sum_{i=1}^{n} P_{i} f_{i}$

where $f_{i}$ is the frequency shift of the $i^{\text {th }}$ spectral point. From the mean Doppler shift, radial winds corresponding to each of the range gates are computed using the Doppler equation.

$v=-c / 2(\Delta f / f)$

Now, if $\mathrm{v}_{1}, \mathrm{v}_{2}$ and $\mathrm{v}_{3}$ are the radial winds corresponding, respectively, to the antenna-north, antenna-west and zenith modes, and $\mathrm{u}, \mathrm{v}$ and $\mathrm{w}$ are the north horizontal, west horizontal and vertical winds, respectively, then the vertically corrected [8] north and west winds are, respectively, given by equations (8) and (9),

i.e. $u=v_{1} \operatorname{cosec} \theta-v_{3} \cot \theta$

and $\quad \mathrm{v}=\mathrm{v}_{2} \operatorname{cosec} \theta-\mathrm{v}_{3} \cot \theta$

and the vertical wind $\mathrm{w}$ is equal to $\mathrm{v}_{3}$

According to the location or placement scheme of antenna, the antenna north and antenna west are rotated clockwise by around $55^{\circ}$ from the geographic north and geographic west, respectively. Therefore, the above-determined horizontal winds are projected on the axis pointing toward the geographic north and geographic west before the final horizontal wind vectors are evaluated.

The minimum period of averaging of each mode for noise as well as signals is $1 \mathrm{~min}$. Therefore, the minimum period required to derive each horizontal wind profile from three radial modes is $6 \mathrm{~min}$. The software is however interactive, and this integration time may be altered.

\section{(f) Timing and Control}

The various timing and control signals are generated by control software in the PC and are output via a PC plug in I/O card and interfaced with different sub-assemblies using suitable interface units. The function of the timing and control subsystem is to generate different control signals in a specific sequence and time relationship for operation of the different switching matrices that regulate the operation of the transmitter, receiver and the antenna system as described above.

The main features of the Acoustic Wind Profiler are summarized in Table 2.

\section{MEASUREMENTS}

The acoustic wind profiler is being used to make measurement of vertical winds, as well as, horizontal winds. Figure 4 (a) depicts the height profiles of 2 minute averaged vertical winds from 0841 hrs to 0923 hrs, taken on $5^{\text {th }}$ May, 2009. The vertical resolution given by the expression $\mathrm{c} \tau / 2$, with $\mathrm{c}$ taken as $350 \mathrm{~m} \mathrm{~s}^{-1}$ and $\tau$ of $200 \mathrm{~ms}$ is $35 \mathrm{~m}$. As per the general convention used, the positive winds are up winds or winds away from the antenna and negative winds are down winds or winds toward the antenna. In the profile, the minimum height of measurement is $70 \mathrm{~m}$ and the maximum height is $455 \mathrm{~m}$. Measurement of higher heights that are not shown did not meet the signal to noise criteria and were therefore rejected. In general, the vertical winds are seen to lie between $-0.5 \mathrm{~m} \mathrm{~s}^{-1}$ and $+0.5 \mathrm{~m} \mathrm{~s}^{-1}$, with exceptions at a few range gates.

Table 2: Main features of the acoustic wind profiler

\begin{tabular}{|c|c|c|}
\hline System frequency & : & $2150 \mathrm{~Hz}$ \\
\hline No. of beams & : & 3 \\
\hline Transmit beam steering & & $19^{\circ}$ (phase) \\
\hline Receive beam steering & & $19^{\circ}$ (time delay) \\
\hline Transmitter power & & $160 \mathrm{~W}$ (elect) \\
\hline Transmit pulse width & : & $200 \mathrm{~ms}$ \\
\hline Acoustic intensity & : & $600 \mathrm{Wm}^{-2}$ \\
\hline Antenna beam width & : & $10^{\circ}$ \\
\hline Receiver gain & : & $110-130 \mathrm{~dB}$ \\
\hline Filter type & : & 8th-order Chebyshev \\
\hline Acoustic antenna & & $\begin{array}{l}\text { Phased array of } 104 \text { Motorola } \\
\text { KSN1025A piezoelectric transducers }\end{array}$ \\
\hline Antenna weight & : & 85 kg (approx.) \\
\hline Antenna transmit efficiency & : & $14.5 \mathrm{PaV}^{-1}$ \\
\hline Antenna receive efficiency & : & $0.7 \mathrm{VPa}^{-1}$ \\
\hline No. of range gates & : & $3-22$ \\
\hline Thickness of each gate & : & 35 m (approx.) \\
\hline Minimum averaging time & : & $6 \min$ \\
\hline Signal processing & : & 1024 pt. FFT \\
\hline Vertical wind speed & : & $0 \pm 4 \mathrm{~m} \mathrm{~s}^{-1}$ \\
\hline Radial wind speed & : & $0 \pm 8 \mathrm{~m} \mathrm{~s}^{-1}$ \\
\hline
\end{tabular}



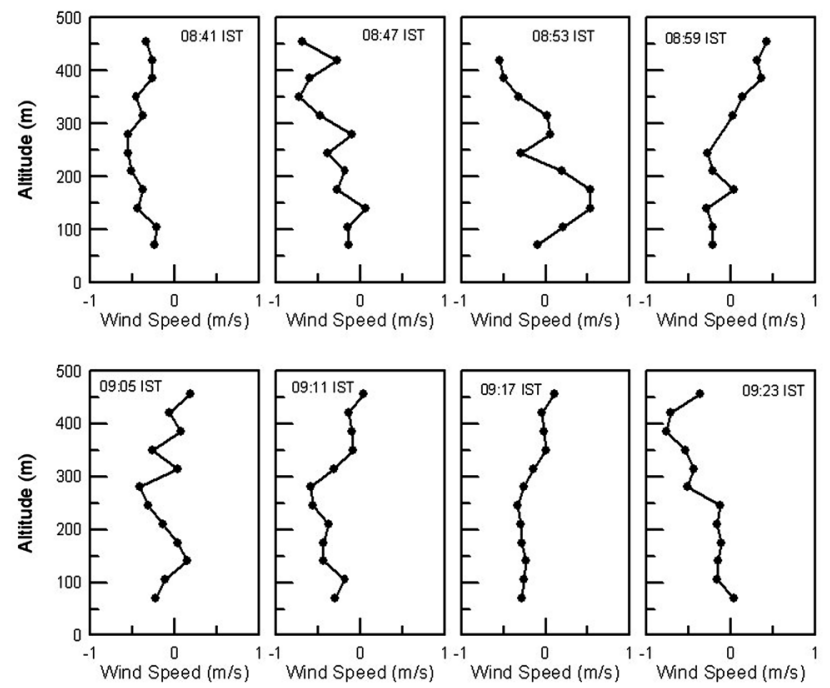

Figure 4: (a) Measurement of the 2-min-averaged vertical winds at an interval of $6 \mathrm{~min}$, from $0841 \mathrm{hrs}$ to $0923 \mathrm{hrs}$, as carried out on 05-05-2009.
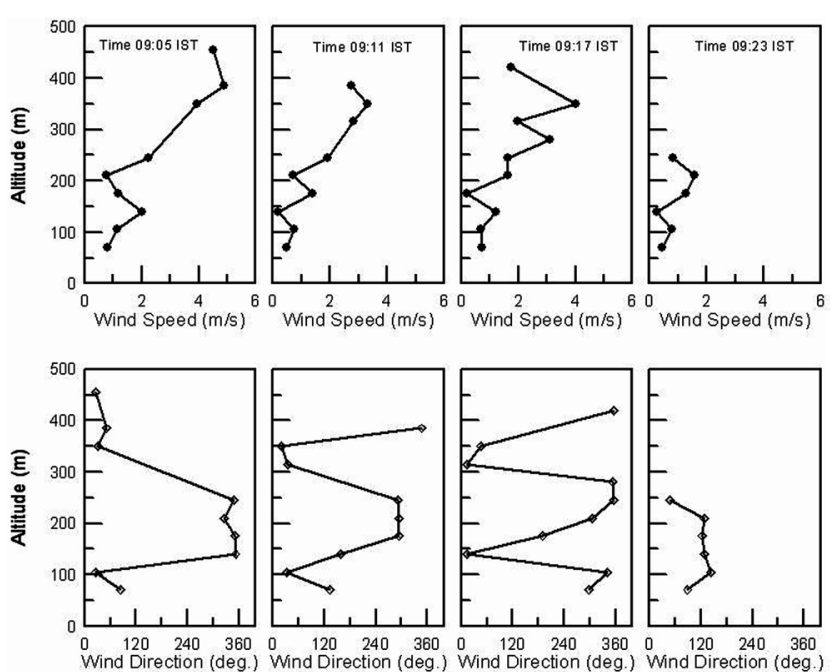

Figure 4: (c) Six-min-averaged values of horizontal wind speed and direction from 0905 hrs to 0923 hrs on 05-05-2009.

Figure 4 (b and c) show the 6 minute averaged height profiles of vertical wind-corrected horizontal wind speed and direction from $0841 \mathrm{hrs}$ to $0929 \mathrm{hrs}$ as taken on $5^{\text {th }}$ May, 2009. As is generally the case, the wind speed is, in general, seen to increase with height. Zero degree represents winds coming from the north. Other directions are degrees clockwise from the north.

\section{APPLICATIONS}

The acoustic wind profiler can find important applications in the earth's boundary layer research, in aviation to observe wind shears in the vicinity of airports for safe landing and take-off of aircrafts, in environmental impact assessment studies and the study of battle field
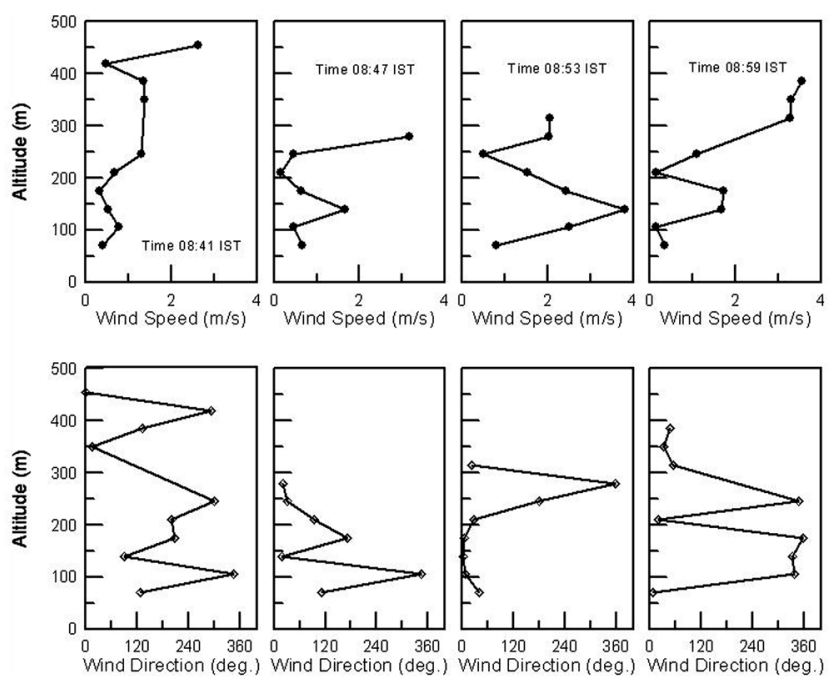

Figure 4: (b) Six-min-averaged values of horizontal wind speed and direction from 0841 hrs to 0859 hrs on 05-052009.

environment for launch of projectiles as well as in wind energy sitting studies.

\section{CONCLUSION}

A system has been developed that provides remote measurement of atmospheric winds in the height range of 70-770 m. However, due to high acoustic noise at the site of its location, it is able to give wind measurements up to $455 \mathrm{~m}$ only. The system can work to its maximum height if the environmental noise at the site is controlled or if it is operated at some semi-urban quieter location.

\section{ACKNOWLEDGMENTS}

The authors are thankful to the Department of Science and Technology for supporting the project under which the present system could be successfully developed. They are also thankful to the chairman and members of the PAMC on MONTCLIM and ICRP and the chairman and members of the Project Review Committee on RASS for their continued help, suggestions and encouragement during the execution of the present work, with special thanks to Dr. P. Sanjeeva Rao for his ever ready help. The authors would also like to thank the director, National Physical Laboratory, and other departments for their continued support in the project. Special thanks are also due to the technical staff and officers of the NPL workshop for their dedicated support.

\section{REFERENCES}

1. R M Khanna, "Remote Measurement Techniques for the Profiling of the Atmospheric Environment: A Review of Atmospheric Wind Measurement", Journal of the Metrology Society of_India, Vol. 12, Nos. 2-4, pp. 131-9, 1997.

2. C G Little, "Acoustic Methods for the Remote Profiling of the Lower Atmosphere", Proc._IEEE, Vol. 57, No. 4, April 1969.

3. A S Monin, "Characteristics of the Scattering of Sound in a Turbulent 
Atmosphere", Sov. Phys.-Acoustics, Vol. 7, pp. 370-3, 1962.

4. M A Kallistratova, "An Experimental Investigation in to the Scattering of Sound in a Turbulent Atmosphere", Dokl.Akad. Nauk. SSR, Vol. 125, No. 1, pp. 69-72, 1959.

5. M A Kallistratova, "Procedure for Investigating Sound Scattering in the atmosphere", Sov. Phys.-Acoustics Vol. 5, pp. 512-4, 1959.

6. S D Danilov, "Simple Method of calibration of Conventional Sodar
Antenna system", International Journal of Remote Sensing, Vol. 15, Issue 2, pp. 307-12, 1994.

7. R M Khanna, O Sharma, and S C Garg, "Design of a High Efficiency Acoustic Phased Array antenna for an Acoustic Wind Profiler", Meteorl.Atmos.Phys ,Vol. 71, No. 1-2, pp. 35-41, 1999.

8. S F Clifford, J C Kaimal, R J Lataitis, and R G Strauch, "Ground based remote profiling in Atmospheric studies: An overview", Proceedings of the IEEE, Vol. 82, No. 3, pp. 313-55, 1994.

\section{AUTHORS}

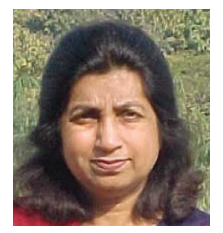

Madhu Bahl obtained her graduation from Institute of Electronics \& Telecommunication Engineers (IETE). She joined the instrumentation group of National Physical Laboratory in 1974 and worked on a number of instruments development projects for various scientific studies. Thereafter she joined Radio and Atmospheric sciences division of NPL as scientist C in 1988. Where she worked on the hardware and software development of Stretched Rohini Satellite Series (SROSS) payload, that was launched successfully by ASLV rocket from Siriharikota. There after she had been engaged in the development of hardware, as well as software in the Deptt. of Science and Technology sponsored project of "Phased Array Acoustic Wind Profiler." Superannuated from NPL in 2011 as Scientist F She has active interest in scientific and technical studies

\section{E-mail: bahlmadhu@nplindia.org}

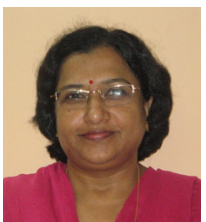

Beena Gupta is presently working in National Physical Laboratory, Delhi as Senior Technical Officer. She has done Diploma in Electronics and Radio Engineering, from Gujarat Technical Board, Ahmadabad in 1981. She joined Physical Research Laboratory Ahmadabad in 1981 and has been involved in fabrication of electronic circuitry. She joined Central Scientific Instruments Organization, Chandigarh in 1983.

E-mail: guptabr@mail.nplindia.org

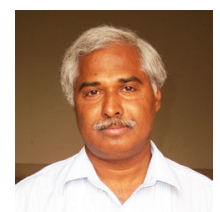

Thomas John is a Postgraduate in Physics and has been working as a scientist in the Radio and Atmospheric Sciences Division of NPL since 1983. He has worked on developing instrumentations for balloon, rocket and satellite experiments as well as ground based instrumentations for atmospheric and ionospheric studies.

E-mail: tjohn@mail.nplindia.org

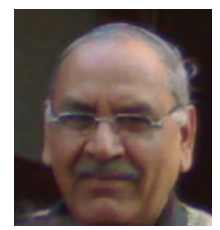

Dhan Singh after graduating in Physics Mr. Dhan Singh joined the communication branch of the Boarder Security Force (BSF) where he was involved in the repair and maintenance of $\mathrm{HF}, \mathrm{VHF}$ and line communication systems. After about 12 years in BSF, he joined the NPL in 1983. In NPL he was involved in the fabrication and testing of various scientific payloads for balloon rocket and satellite experiments as well as ground based instrumentations for atmospheric research till his retirement in 2008.

\section{E-mail: dschaunal@gmail.com}

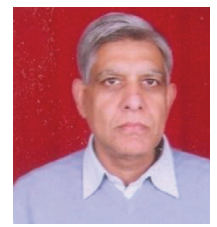

Omkar Sharma obtained his masters in physics from University of Delhi in the year 1973. He joined the Acoustics Group of National Physical Laboratory in December 1973 and worked on several projects. Presently he is Scientist $G$ and leading the Acoustic Group working on realization of Primary standards of Acoustics and Calibration of secondary and working standards against the primary standards. To ensure international harmonization with participation in key comparisons at CCAUV/APMP level. To establish a traceable calibration measurement service linked by an unbroken chain of comparison to the primary standard of measurement. Noise/vibration measurements, analysis and control. Consultancy in Architectural Acoustics and R\&D in Applied Acoustics.

E-mail: osharma@mail.nplindia.org

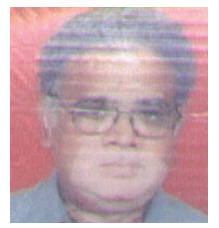

S. C. Garg after obtaining M.Sc. Tech (Electronics) in 1969 from BITS, Pilani, Mr.Garg joined NPL as a scientist and contributed immensely to the design and development of instrumentations for atmospheric studies. He was the leader of the group in NPL for the development of scientific payloads for balloon, rocket and satellite experiments of upper atmospheric and ionospheric research. He was the principal investigator for the first Indian Aeronomy satellite experiment in which NPL designed and developed the Retarding Potential Analyzer (RPA) payload flown aboard the Indian satellites SROSS-C \&C2. He served as Head of the Radio and Atmospheric Sciences Divisions of NPL from 2000 - 2006. After his retirement in 2006, he continued his research activities as an emeritus scientist of CSIR.

E-mail: gargnpl@gmail.com

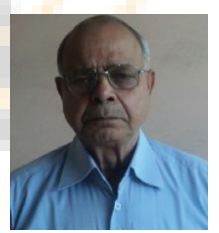

Ravi M. Khanna obtained his DMIT (Electronics \& Telecommunication Engg.) from MIT, Tamilnadu in 1969 after his B.Sc. from Jammu \& Kashmir University. He joined DRDO in 1969 and worked in various developmental projects in DRDO laboratories i.e. (HRPU) DEAL, Dehradun and SSPL, Delhi from 1969 to 1977. He joined the National Physical Laboratory as Scientist B in 1977, where, initially, he worked on the development of rocket pay loads for the study of the ionosphere and participated in an experiment conducted on board Rohini-560 rocket from Sriharikota. From 1983 onwards, he had been working on the development of systems for research in the boundary layer atmosphere, where he initially developed a Complex Covariance Doppler Sodar to measure atmospheric vertical winds on which one of his publications was conferred with Indian Meteorological Society's J. Das Gupta award. Thereafter he worked on the design, development and studies of Phased Array Acoustic Antennae and also developed an Acoustic Radar Equation based on these studies. From 1997 to 2002, he lead a scientific team to successfully develop a Phased Array Acoustic Wind Profiler under a Department of Science \& Technology sponsored project. He was also honorary faculty member BITS Pilani in 1991-93 and taught Digital Systems and Microprocessors in M.S. Physical Sciences course of NPL- BITS collaborative program. He has presented his research in a number of national and international conferences in India and abroad and has about twenty one publications in national and international journals besides co-authoring a course book in electronic science for Indra Gandhi National Open University. Superannuated from NPL in 2003 as Scientist F, he carries an active interest in scientific and technical pursuits and is member of Acoustical Society of India, Indian Meteorological Society, Metrology Society of India and member Indian Association for Air Pollution Control.

E-mail: rkravikhanna@gmail.com 